\title{
Article \\ Ag Surface and Bulk Segregations in Sputtered ZrCuAlNi Metallic Glass Thin Films
}

\author{
Michael K. Steinhoff ${ }^{1,+}{ }^{+}$, Damian M. Holzapfel ${ }^{1}$ (D), Soheil Karimi Aghda ${ }^{1}$ (D), Deborah Neuß ${ }^{1}$, Peter J. Pöllmann ${ }^{1}$, \\ Marcus Hans ${ }^{1} \mathbb{D}$, Daniel Primetzhofer ${ }^{2}\left(\mathbb{D}\right.$, Jochen M. Schneider ${ }^{1}$ and Clio Azina ${ }^{1, *(D)}$
}

1 Materials Chemistry, RWTH Aachen University, Kopernikusstraße 10, D-52074 Aachen, Germany; michael.steinhoff@rwth-aachen.de (M.K.S.); holzapfel@mch.rwth-aachen.de (D.M.H.); karimi@mch.rwth-aachen.de (S.K.A.); neuss@mch.rwth-aachen.de (D.N.); poellmann@mch.rwth-aachen.de (P.J.P.); hans@mch.rwth-aachen.de (M.H.); schneider@mch.rwth-aachen.de (J.M.S.)

2 Department of Physics and Astronomy, Uppsala University, Lägerhyddsvägen 1, S-75120 Uppsala, Sweden; daniel.primetzhofer@physics.uu.se

* Correspondence: azina@mch.rwth-aachen.de; Tel.: +49-241-8025997

† Present address \& email: Fraunhofer Institute for Laser Technology ILT, Steinbachstraße 15, D-52074 Aachen, Germany; michael.steinhoff@ilt.fraunhofer.de.

Citation: Steinhoff, M.K.; Holzapfel, D.M.; Karimi Aghda, S.; Neuß, D.;

\section{Pöllmann, P.J.; Hans, M.;}

Primetzhofer, D.; Schneider, J.M.; Azina, C. Ag Surface and Bulk Segregations in Sputtered ZrCuAlNi Metallic Glass Thin Films. Materials 2022, 15, 1635. https://doi.org/ $10.3390 / \mathrm{ma} 15051635$

Academic Editors: Nikolas J. Podraza, Dameng Liu, Jordi Faraudo and Konda Gokuldoss Prashanth

Received: 28 December 2021 Accepted: 18 February 2022 Published: 22 February 2022

Publisher's Note: MDPI stays neutral with regard to jurisdictional claims in published maps and institutional affiliations.

Copyright: (C) 2022 by the authors. Licensee MDPI, Basel, Switzerland. This article is an open access article distributed under the terms and conditions of the Creative Commons Attribution (CC BY) license (https:// creativecommons.org/licenses/by/ $4.0 /)$.
Abstract: We report on the formation of Ag-containing $\mathrm{ZrCuAlNi}$ thin film metallic glass (nano)composites by a hybrid direct-current magnetron sputtering and high-power pulsed magnetron sputtering process. The effects of Ag content, substrate temperature and substrate bias potential on the phase formation and morphology of the nanocomposites were investigated. While applying a substrate bias potential did not strongly affect the morphological evolution of the films, the Ag content dictated the size and distribution of Ag surface segregations. The films deposited at low temperatures were characterized by strong surface segregations, formed by coalescence and Ostwald ripening, while the volume of the films remained featureless. At higher deposition temperature, elongated Ag segregations were observed in the bulk and a continuous Ag layer was formed at the surface as a result of thermally enhanced surface diffusion. While microstructural observations have allowed identifying both surface and bulk segregations, an indirect method for detecting the presence of $\mathrm{Ag}$ segregations is proposed, by measuring the electrical resistivity of the films.

Keywords: thin film metallic glass; nanocomposites; segregation; electrical resistivity

\section{Introduction}

Nanocomposites are being developed for a variety of applications as they allow combining the properties of two or more constituents. Nanoscale reinforcements, such as nanoparticles (NPs), nanowires, etc., which possess unique physicochemical properties, can provide novel functionalities and/or enhance the properties of the matrix. Metallic reinforcements have been embedded in a variety of matrices, the choice of which often depends on the targeted applications [1-3]. In fact, metallic reinforcements are often considered as they are ductile and promote recrystallization in ceramics, which leads to finer microstructures, and in turn, impact the mechanical properties [4,5]. More specifically, Ag has often been employed not only because of its chemical inertness but also because of its biocidal properties [6,7] which make it suitable for bio-related applications.

Several studies have reported the formation of Ag-containing nanocomposites by sputtering. For example, TiSiN:Ag [8] and TiCN:Ag [9] nanocomposites were produced by reactively sputtering from a Ti target which contained either Si and Ag, or just Ag pellets, respectively. Domingues and coworkers followed a similar approach to produce AlN:Ag nanocomposites from an Al target comprising a variable number of Ag pellets [10]. With this approach, the number of Ag pellets influenced the Ag content of the films, however, 
there is little flexibility over the deposition rate of the films as all metallic constituents are operated in the same conditions. Co-sputtering from independent targets, allows for better control over the deposition rates of each constituent, while also varying Ag contents by adjusting the targets powers [11-13].

Regardless of the sputtering approach, common features observed in such films were Ag segregations. The presence of these segregations was influenced by process parameters such as the power applied to the Ag target, and therefore, the Ag content $[4,14,15]$, the substrate temperature [16] as well as the bias potential during deposition $[11,15,17,18]$. The surface segregations were a result of Ag diffusion towards the surface followed by coalescence. The surface and bulk diffusion of Ag atoms can be enhanced with respect to the process parameters listed above. Additionally, highly energetic processes such as high-power pulsed magnetron sputtering (HPPMS) may also contribute to enhanced surface diffusion [15]. Few studies also reported Ostwald ripening as a mechanism for segregation $[15,19]$. Cloutier et al. used a hybrid method based on plasma enhanced chemical vapor deposition (PECVD) and very low frequency sputtering, to produce Agcontaining diamond-like carbon (DLC) films. The authors reported the presence of Ag clusters on the surface of the DLC as well as within the bulk. In their case, a bimodal size distribution was detected on the surface which supported the occurrence of Ostwald ripening in tandem with diffusion/coalescence processes [19].

The formation of Ag segregations has been reported for several cases of ceramic matrices [2], owing to the immiscibility and lack of reactivity of Ag and the matrices. However, little information can be found on Ag segregation in metallic matrices. In fact, incorporation of $\mathrm{Ag}$ in metallic systems is often in the aim of producing novel metallic glass (MG) compositions, often for antibacterial applications [20-22]. While efforts are concentrated on identifying the composition range up to decomposition and/or crystallization [23-25], the mechanisms of Ag segregation in thin film MGs have not been widely discussed.

In this work, the surface and bulk segregation of $\mathrm{Ag}$ in $\mathrm{ZrCuAlNi}$ metallic glass thin films is presented and discussed. The films were obtained by a hybrid direct current magnetron sputtering (DCMS)/HPPMS co-sputtering process. The effects of substrate temperature, bias potential and Ag content on the microstructure of the produced films are presented and the mechanisms responsible for surface and bulk segregation are discussed. Finally, electrical resistivity measurements were carried out in order to verify this method for identifying the presence of bulk segregations.

\section{Materials and Methods}

Ag-containing metallic glasses were deposited using co-sputtering from an elemental Ag target ( $20 \mathrm{~mm}, 99.99 \%$, M\&K GmbH), operated in HPPMS, and two identical $\mathrm{Zr}_{60} \mathrm{Cu}_{25} \mathrm{Al}_{10} \mathrm{Ni}_{5}$ powder metallurgical composite targets, which will be referred to as MG in the text (Ø $20 \mathrm{~mm}$, PLANSEE Composite Materials GmbH, Lechbruck Am See, Germany), operated in DCMS. Pure thin film MGs were also deposited, for comparison, in DCMS mode. An ultra-high vacuum (UHV) chamber, with a base pressure below $4 \times 10^{-6} \mathrm{~Pa}$ and a target-to-substrate distance of $100 \mathrm{~mm}$, was used to deposit the MG and Ag-containing MGs on $10 \times 10 \mathrm{~mm}^{2}$ c-cut sapphire, $\mathrm{Al}_{2} \mathrm{O}_{3}(0001)$ substrates which were used as delivered, i.e., with no pre-cleaning. All targets were operated in constant power mode, while the substrates were rotated to ensure a homogeneous elemental distribution. Ar (99.9999\%) was used as sputtering gas with a pressure $0.55 \mathrm{~Pa}$. Figure 1 represents the target positions in the UHV chamber, and more information on the geometry within the chamber can be found elsewhere [26]. HPPMS and DCMS were performed using a Melec SIPP2000USB-10-500-S pulser combined with a 1.5 kW ADL GP 15/1000 DC power supply and an Advanced Converters PMP-1 DC power supply, respectively. 


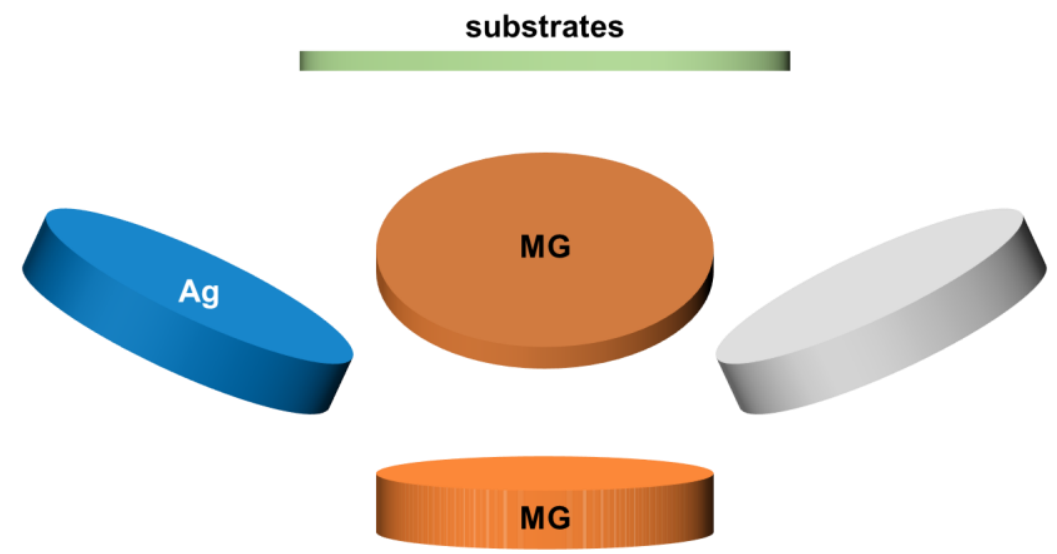

Figure 1. Schematic of target positioning in the ultra-high vacuum (UHV) chamber. The grey disc, on the right-hand side, represents the fourth magnetron of the chamber which was not used during depositions.

The parameters that were varied during depositions are summarized in Table 1 . The Ag content was controlled by changing the applied power on the MG targets, while keeping the Ag one constant. For example, for the high Ag content (30 at.\%) the metallic glass targets were operated at $22 \mathrm{~W}$, each. For lower Ag contents, the target power on the metallic glass targets were increased. The powers used were decided after test depositions were carried out to determine the Ag content and deposition rate at each condition. Then, the sputtering times were adapted in order to deposit $\sim 1 \mu \mathrm{m}$-thick films. Here too, test depositions were carried out to adapt the deposition durations. Indeed, for $30 \mathrm{at} \% \mathrm{Ag}$, the operating power of the MG targets were decreased, which resulted in lower deposition rates. Therefore, the deposition duration was increased in order to deposit $\sim 1 \mu \mathrm{m}$-thick film (cross-sections measured in the focused ion beam microscope).

Table 1. Deposition parameters used to deposit the pure metallic glasses (MGs) and the Ag-containing thin films.

\begin{tabular}{lcc}
\hline Deposition Parameters & Zr60Cu25Al10Ni5 & Ag \\
\hline Sputtering mode & DCMS * & HPPMS ${ }^{* *}$ \\
Target power $(\mathrm{W})$ & 22-100/target & 40 \\
Duty cycle $(\%)$ & 0.25 \\
$\mathrm{t}_{\text {on }} / \mathrm{t}_{\text {off }}$ & & $80 / 31,920$ \\
Peak current density $\left(\mathrm{A} / \mathrm{cm}^{2}\right)$ & $0.18-0.35$ \\
Substrate temperature $\left({ }^{\circ} \mathrm{C}\right)$ & \\
Substrate bias potential $(\mathrm{V})$ & $60,200,400$ \\
${ }^{*}$ Direct-current magnetron sputtering; ${ }^{* *}$ High power pulsed magnetron sputtering; ${ }^{1}$ Floating voltage of $\sim 10 \mathrm{~V}$.
\end{tabular}

Structural properties of the deposited films were characterized by means of X-ray diffraction (XRD), using a Bruker AXS D8 Discover X-ray diffractometer with an integrated general area detector diffraction system (GADDS), with $\mathrm{Cu}$ radiation. An incident angle of $15^{\circ}$ was used, while diffractograms were collected within the $2 \theta$ range of 20 to $70^{\circ}$. X-ray reflectivity (XRR) was used to determine the density of the pure MG films. The measurements were carried out in a Panalytical Empyrean diffractometer with $\mathrm{Cu}$ radiation and GenX was used for fitting [27].

Ion beam analysis was carried out at the Tandem Laboratory of Uppsala University. Chemical composition depth profiling was done through time-of-flight elastic recoil detection analysis (ERDA). We directed $36 \mathrm{MeV}^{127} \mathrm{I}^{8+}$ projectiles at the specimen with incidence and exit angles of $22.5^{\circ}$ with respect to the sample surface, while the angle between the ion beam and detector was $45^{\circ}$. Since $\mathrm{Cu}$ and Ni cannot be straightforwardly differentiated due to similar mass, their ratio was determined more accurately by Rutherford backscattering spectrometry (RBS) using $2 \mathrm{MeV}^{4} \mathrm{He}^{+}$primary ions detected at a scattering angle of $170^{\circ}$. 
RBS also further improves the accuracy in the deduced Zr:Ag ratio. A detailed discussion of accuracies achievable in a combination of different ion beam analytical methods for systems based on multiple transition metals can be found elsewhere [28]. ERDA and RBS data analysis was undertaken using CONTES [29] and SIMNRA [30], respectively.

Chemical compositions were measured with a JEOL JSM-6480 scanning electron microscope equipped with an EDAX Genesis 2000 energy-dispersive X-ray spectroscopy (EDX) detector. Measurements were carried out with an acceleration voltage of $7 \mathrm{kV}$ and a magnification of $4000 \times$ under a high vacuum. EDX spectra were quantified based on two reference samples which have been measured by ion beam analysis: $\mathrm{Zr}_{50} \mathrm{Cu}_{26} \mathrm{Al}_{10} \mathrm{Ni}_{7} \mathrm{O}_{7}$ was used for determining the $\mathrm{Zr}-\mathrm{Cu}-\mathrm{Al}-\mathrm{Ni}$ ratios, while the $\mathrm{Ag}$ and $\mathrm{O}$ reference concentrations were obtained from a $\mathrm{Zr}_{43} \mathrm{Cu}_{20} \mathrm{Al}_{7} \mathrm{Ni}_{8} \mathrm{Ag}_{17} \mathrm{O}_{5}$ film. Scanning electron microscopy (SEM) micrographs were captured with a Hitachi TM4000Plus Tabletop microscope using a backscattered electron (BSE) detector.

Thin lamellae and atom probe tomography (APT) specimens were prepared in a FEI Helios NanoLab dual-beam focused ion beam (FIB) microscope, using $\mathrm{Ga}^{+}$ions at $30 \mathrm{kV}$ acceleration voltage. Scanning transmission electron microscopy (STEM) was carried out on the lamellae using a STEM III detector in high-angle annular dark field (HAADF) mode. Furthermore, EDX line scans (quantification without standard) were carried out with an EDAX Octane Elect EDX detector.

Three-dimensional spatially-resolved chemical composition analysis at the nanometer scale was carried out by atom probe tomography (APT) using a CAMECA local electrode atom probe $4000 \times \mathrm{HR}$. Laser-assisted evaporation was employed with $50 \mathrm{pJ}$ laser pulse energy, $125 \mathrm{kHz}$ laser pulse frequency, $60 \mathrm{~K}$ base temperature and $0.5 \%$ average detection rate. Data analysis was undertaken using IVAS 3.8.0.

The resistivity of the films was measured using a Van der Pauw setup containing four contacts which were pressed onto the sample surface. A Keithley 2611B System SourceMeter Unit (SMU) was used to provide the current of $5 \mathrm{~mA}$ and to measure the resulting voltage. An average of eight sheet resistance values was obtained for all possible configurations of the four contacts, in order to exclude the effect of contact resistance, and repeated 20 times, resulting in one resistivity value. The procedure was repeated three times by removing the contacts and placing them on the surface again. The electrical resistivity was then deduced by considering the thicknesses of each film. One resistivity datapoint given corresponds to the average of the three measurements and the error bars correspond to the standard deviation.

\section{Results and Discussion}

Bönninghoff et al. discussed the growth of a $\mathrm{Zr}_{60} \mathrm{Cu}_{25} \mathrm{Al}_{10} \mathrm{Ni}_{5}$ thin-film metallic glass (TFMG) produced by HPPMS and DCMS, and have reported differences in the microstructure and density of the films produced [31]. Considering the material system of the pure MG being identical to that presented in this work, albeit from a target produced by a different manufacturer, films were reproduced in DCMS and HPPMS modes in order to confirm that the microstructures produced were featureless. It appeared that both the films deposited at 60 (not shown) and $200{ }^{\circ} \mathrm{C}$, resulted in featureless microstructures, as seen in Figure 2a, regardless of the deposition mode. The density of the film deposited here with DCMS was $6.8 \mathrm{~g} / \mathrm{cm}^{3}$, which is as dense as the film deposited with HPPMS in [31]. Furthermore, the chemical composition was homogeneous throughout the thickness of the films as determined by ERDA depth profiling and EDX line scans (see Figure 2b). Therefore, the MG targets were operated in DCMS to avoid decreased deposition rates related to the HPPMS mode [32]. 
(a)

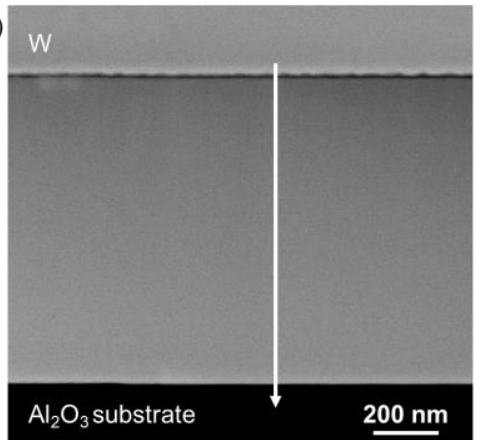

Chemical Composition (at \%)

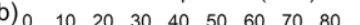

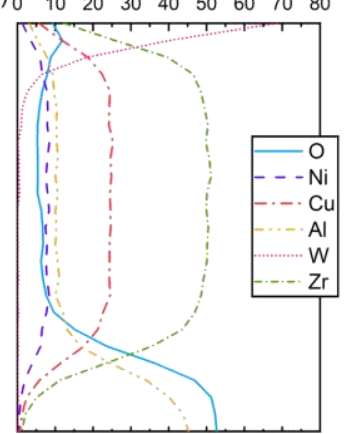

(c)

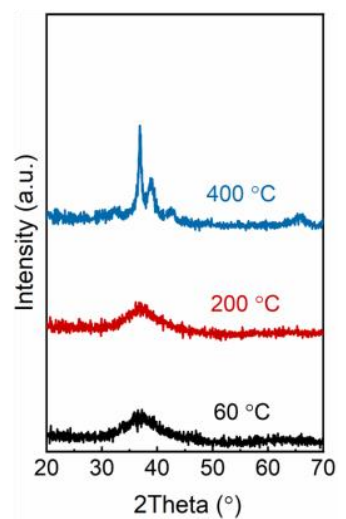

Figure 2. (a) High-angle annular dark field scanning transmission electron microscopy (HAADF STEM) image of the $\mathrm{ZrCuAlNi}$ film deposited at $200{ }^{\circ} \mathrm{C}$ with direct current magnetron sputtering (DCMS) and (b) energy-dispersive X-ray spectroscopy (EDX) line scan measured along the arrow represented in (a). (c) X-ray diffraction of thin-film metallic glasses (TFMGs) deposited at 60, 200 and $400{ }^{\circ} \mathrm{C}$.

Figure 2c gathers the X-ray diffractograms of the TFMGs deposited at 60, 200 and $400{ }^{\circ} \mathrm{C}$. The motivation behind the deposition at a substrate temperature of $60^{\circ} \mathrm{C}$ was to avoid possible local heating [15], induced by ion bombardment, during film growth. While the films deposited at 60 and $200{ }^{\circ} \mathrm{C}$ did not show any detectable peaks, the films deposited at $400{ }^{\circ} \mathrm{C}$ were at least in part crystalline. Therefore, they were not considered further. Furthermore, the films deposited with substrate bias potential were still $\mathrm{X}$-ray amorphous at both 60 and $200{ }^{\circ} \mathrm{C}$, while neither the microstructure nor the elemental distribution appeared to be affected.

A hybrid DCMS/HPPMS process was used to deposit the Ag-containing TFMGs in order to promote densification of the films and allow deposition of limited amounts of Ag. The X-ray diffraction patterns of the resulting films deposited without substrate bias at 60 and $200{ }^{\circ} \mathrm{C}$ are reported in Figure 3a,b, respectively. While the Ag-containing TFMGs deposited at $60^{\circ} \mathrm{C}$ were still X-ray amorphous, one can notice a shift of the broad hump towards higher $2 \theta$ angles with increasing $\mathrm{Ag}$ content as the films become less $\mathrm{Zr}$-rich. The films containing 15 and 30 at. $\%$ of $\mathrm{Ag}$, which were deposited at $200{ }^{\circ} \mathrm{C}$, exhibited peaks corresponding to cubic Ag (ICDD: 00-004-0783). The peaks observed in Figure 3b suggest Ag segregation. While the peaks are of low intensity at 15 at.\%, they become more prominent with increasing $\mathrm{Ag}$ content, indicating that more $\mathrm{Ag}$ has segregated and that the amount of metallic Ag has increased. The XRD patterns of the corresponding films deposited with substrate bias potential (not shown here) were comparable to those deposited without bias. The detection of metallic Ag at high substrate temperature and high Ag contents indicates limited metastable solubility of Ag in the MG, and the simultaneous growth of Ag and MG. The limited solubility of Ag in the MG could be assigned to the positive heat of mixing of $\mathrm{Ag}$ and $\mathrm{Cu}$ [33,34]. Aside from that binary, Ag-Ni [35] also exhibits a positive heat of mixing while those of the remaining binaries (i.e., Ag-Zr [33] and $\mathrm{Ag}-\mathrm{Al}[36]$ ) are negative. Among the metallic glass constituents, all binaries possess a negative mixing enthalpy [37,38], except $\mathrm{Cu}-\mathrm{Ni}$ [39]. Therefore, with increasing Ag contents, one may expect Ag segregation as kinetic limitations are overcome by enabling surface diffusion, as well as bulk diffusion due to positive enthalpy of mixing of Ag with two out of four MG constituents. 

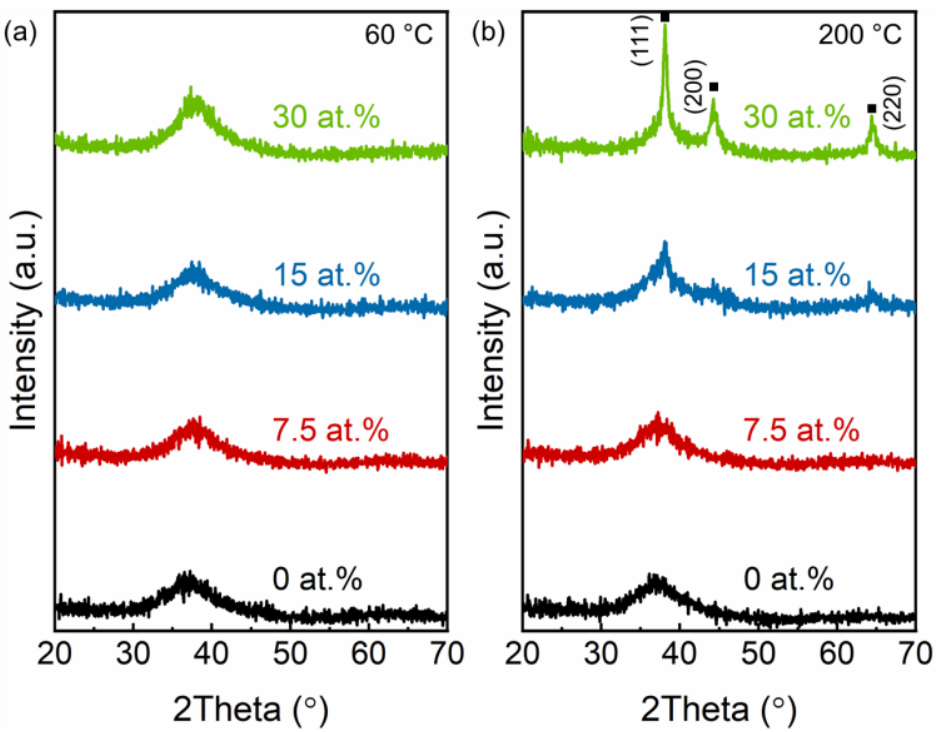

Figure 3. X-ray diffractograms (XRD) of TFMG and Ag-containing TFMGs deposited without substrate bias potential at (a) 60 and (b) $200{ }^{\circ} \mathrm{C}$.

The effect of the substrate bias potential was observed on the surface morphologies of the Ag-containing TFMGs. On the one hand, the $60^{\circ} \mathrm{C}$ depositions under floating potential showed the formation of nanoparticles (NPs) at the surface of the films containing more than 15 at.\% of Ag (Figure $4 \mathrm{a}-\mathrm{c}$ ). Indeed, at 15 at.\% one can observe a bimodal size distribution where the larger NPs are $\sim 42 \mathrm{~nm}$, while the smaller ones are $\sim 25 \mathrm{~nm}$. The size distribution becomes somewhat homogeneous $(\sim 35 \mathrm{~nm})$ as the NPs become more numerous when the Ag content rises to 30 at. $\%$. On the other hand, $-80 \mathrm{~V}$ bias potential led to the formation of NPs already at 7.5 at.\% suggesting that the mobility of Ag atoms increased with bias (Figure $4 \mathrm{~d}$ ). The NP size for 7.5 at. $\%$ of Ag averaged at $\sim 43 \mathrm{~nm}$ and then decreased with increasing Ag content to $20 \mathrm{~nm}$ for 15 at.\% of Ag. At 30 at.\% two observations could be made. First, the size distribution of the NP was bimodal with the largest NPs averaging $\sim 22 \mathrm{~nm}$, while the smallest ones averaged $\sim 10 \mathrm{~nm}$. Second, an increase in substrate bias potential caused an increase in surface roughness as shown in Figure $4 \mathrm{f}$.

The NP formation is attributed to the low metastable solubility of Ag in the MG, causing Ag to migrate to the surface and to grow initially by coalescence and then by Ostwald ripening. Indeed, the NP size distribution, in Figure $4 \mathrm{~b}$ in particular, is not homogeneous but rather composed of larger NPs surrounded by smaller ones. These observations are, according to Cloutier et al. [19] and Mulligan et al. [40], consistent with Ostwald ripening. Additionally, it is interesting to note that the unbiased samples with 15 and 30 at. $\% \mathrm{Ag}$, deposited at $60^{\circ} \mathrm{C}$ were covered with NPs without additional energy contributions, indicating that segregation is mostly caused by higher $\mathrm{Ag}$ concentration, as supported by [18].

When comparing the 7.5 at.\% Ag-containing films deposited at $200{ }^{\circ} \mathrm{C}$ with and without bias (Figure 4g,j), one can clearly see that the bias-induced ion bombardment tends to provide sufficient energy for Ag to migrate and to segregate at the surface. The large grains detected are faceted, similar to what Mulligan et al. observed after annealing $\mathrm{CrN}: \mathrm{Ag}$ at temperatures comprised between 425 and $625^{\circ} \mathrm{C}$ in [40]. With increasing Ag content, a continuous, polycrystalline Ag-rich layer is formed on top of the MG (Figure $4 \mathrm{~h}, \mathrm{i}, \mathrm{k}, \mathrm{l}$ ). This layer is partly responsible for the Ag peaks indexed in Figure 3b. The formation of such layer is also due to the enhanced mobility of Ag atoms, provided by the increased substrate temperature combined with the slower deposition rates that accompany the high Ag-content depositions. Indeed, longer depositions times allowed for Ag to diffuse, segregate and form the Ag layer. Similar observations were made by Hu et al. who reported 
the heat-induced formation of a dense Ag layer on the coating surface of a YSZ:Ag-Mo nanocomposite coating [41].
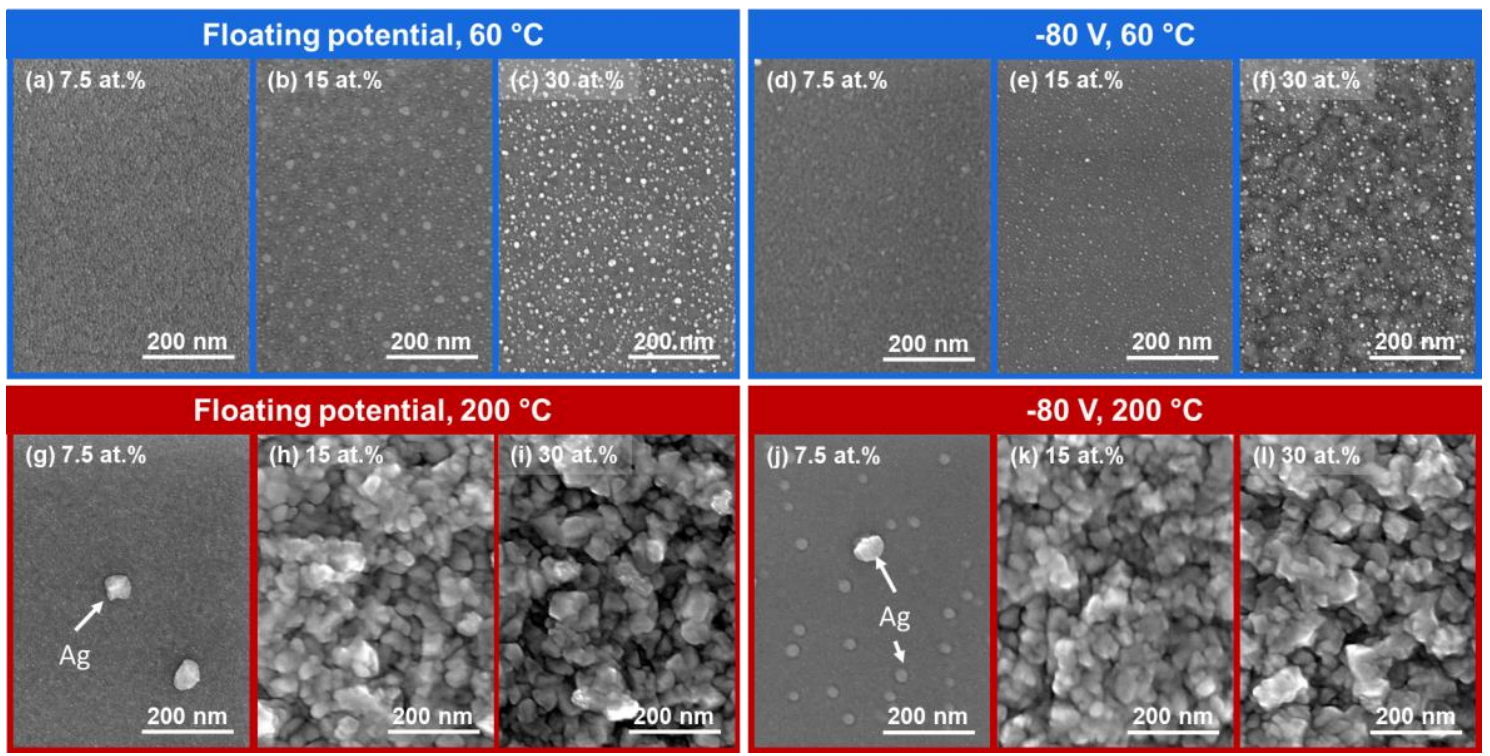

Figure 4. Scanning electron microscopy (SEM) surface micrographs of films of different Ag contents deposited at $(\mathbf{a}-\mathbf{c})$ floating potential; $60{ }^{\circ} \mathrm{C},(\mathbf{d}-\mathbf{f})-80 \mathrm{~V} ; 60^{\circ} \mathrm{C},(\mathbf{g}-\mathbf{i})$ floating potential; $200{ }^{\circ} \mathrm{C}$, and $(\mathrm{j}-1)-80 \mathrm{~V} ; 200{ }^{\circ} \mathrm{C}$.

While Ag was detected at the surface of most films either in the form of NPs or in the form of polycrystalline layers, the presence of Ag segregations throughout the film thickness remained a possibility. Therefore, cross-section lamellae of films were examined by STEM in HAADF mode, while the compositions along the film thicknesses were collected using EDX (Figure 5). The films deposited at $60{ }^{\circ} \mathrm{C}$, of all Ag contents, with and without bias exhibited the same featureless cross-section microstructure and homogeneous composition as the film shown in Figure 5a. The lack of features, therefore, supports the preferential surface segregation at lower substrate temperatures, regardless of Ag content. However, the Agcontaining films with $\geq 15$ at. $\%$ of $\mathrm{Ag}$ deposited at $200{ }^{\circ} \mathrm{C}$ were characterized by continuous Ag layers on the surface (similar thicknesses for both Ag contents) and Ag segregations in the volume of the TFMG, confirming the formation of a dual-phase composite film of Ag (light grey) embedded in a featureless MG matrix (dark grey). Indeed, these films exhibited elongated Ag agglomerates in the volume of the TFMGs (Figure 5c,e) and the size and number increased with increasing Ag content. This finding is similar to observations made by Mulligan et al. and Incerti et al. in the case of CrN:Ag nanocomposites [40,42], according to whom the lamellar shape can be attributed to continuous growth on top of the elongated agglomerates. As shown from the line scans in Figure 5d,f, these agglomerates are Ag-based, indicating that they could also contribute to the Ag peaks indexed in the XRD pattern in Figure 3. Therefore, there seems to be a threshold Ag content which is needed to start the agglomeration within the bulk of the film at higher temperatures. The formation of the Ag segregations was independent of bias potential, indicating that it was directly related to the substrate temperature. In fact, as mentioned previously the substrate temperature is responsible for the enhanced surface diffusion of Ag species.

The overall compositions of the produced films are provided in Table 2. The compositions are fairly comparable regardless of deposition temperature or substrate bias potential. Significant changes occur when varying the Ag content. With increasing Ag content, $\mathrm{Cu}$ and $\mathrm{Zr}$ appear to decrease the most. At 30 at.\% Ag all MG constituents decrease as the $\mathrm{Zr}$ to $\mathrm{Ag}$ ratio approaches 1 . The films were characterized by $\sim 3$ to 9 at. $\% \mathrm{O}$. 


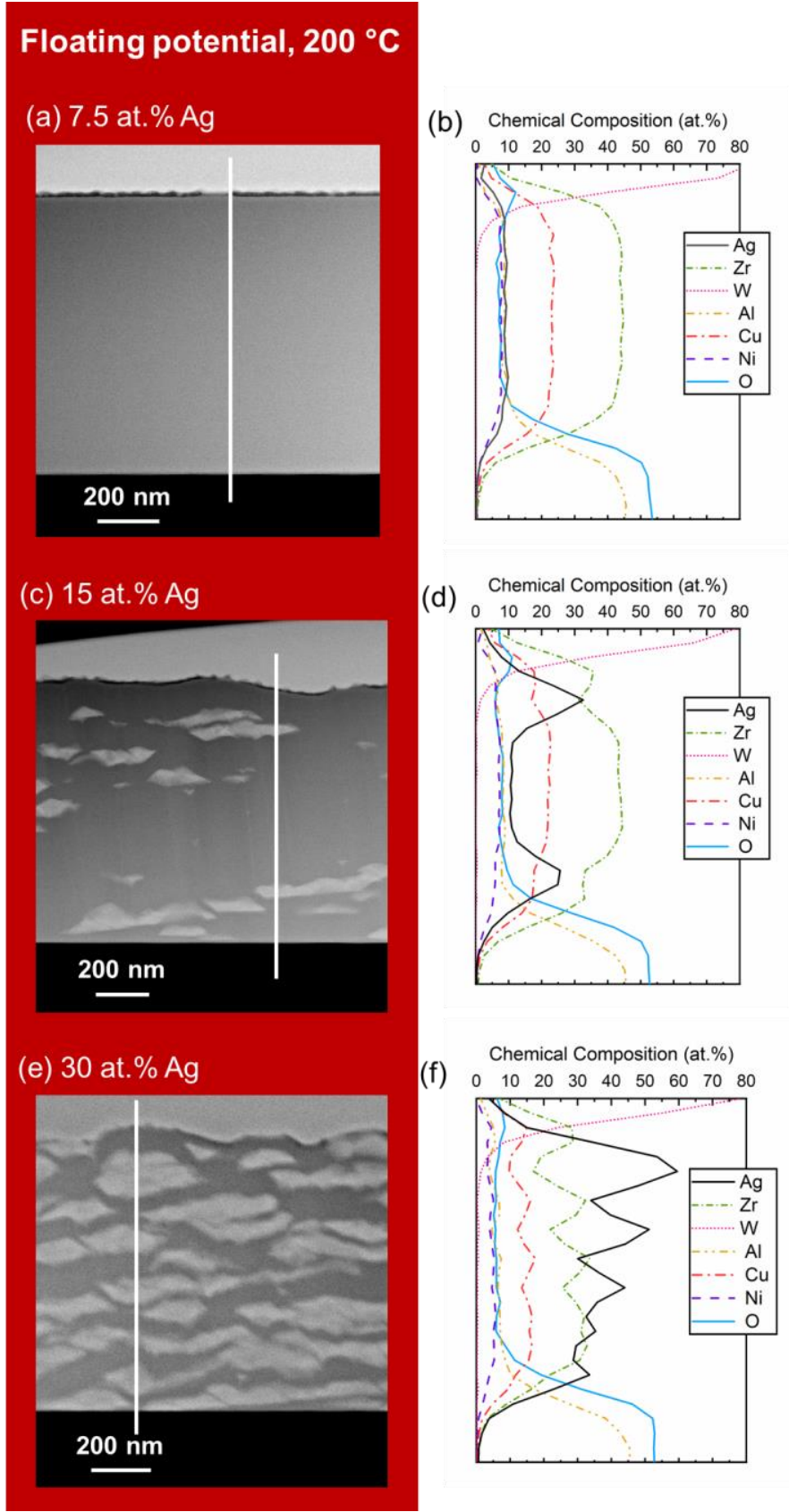

Figure 5. STEM cross-sectional HAADF images and corresponding EDX line scans of the films deposited at $200{ }^{\circ} \mathrm{C}$, under floating potential, containing (a,b) 7.5, (c,d) 15, and (e,f) 30 at.\% Ag.

In order to examine the effect of non-segregated $\mathrm{Ag}$ on the elemental distribution of the MG deposited at low temperature, APT was performed on specimens retrieved from unbiased TFMGs deposited at $60{ }^{\circ} \mathrm{C}$, without and with $30 \mathrm{at} . \% \mathrm{Ag}$. The reconstructions of the atomic positions and the concentration profiles corresponding to each film are shown in Figure 6. The chemical composition, at the nanometer scale, of the pure MG is homogeneous and characterized by a random elemental distribution, as seen in Figure 6a. Furthermore, the concentration profile in Figure $6 \mathrm{~b}$ is representative of the composition of the target material (oxygen content $<0.3$ at.\%). The insertion of $\mathrm{Ag}$ has a non-negligible effect on the elemental distribution in the metallic glass. Indeed, segregations can be seen in Figure $6 c$, while the concentration profiles of all elements, in Figure $6 \mathrm{~d}$, fluctuate significantly. The fluctuations are in agreement with observations made by Oh et al. as one may notice that in the Ag-depleted regions, the Cu concentration is higher [43]. More importantly, in 
some of these regions, the $\mathrm{Cu}$ concentration exceeds the nominal 25 at.\% of the original $\mathrm{MG}$, indicating regions of $\mathrm{Cu}$ clustering within the film. The distribution of $\mathrm{Zr}$ is also significantly affected and seems to fluctuate in a similar manner as $\mathrm{Cu}$, although further investigations would be required to conclude on its behavior. Although present in lower contents, the $\mathrm{Al}$ and $\mathrm{Ni}$ profiles are also impacted by the Ag addition. It is important to note that the film considered with 30 at.\% Ag is actually no longer $\mathrm{Zr}$-rich, as the $\mathrm{Zr}$ to $\mathrm{Ag}$ ratio is close to 1, according to EDX measurements in Table 2 and supported by the broad band shift observed in Figure 3a. Therefore, we suggest the formation of a new Ag- and Zr-rich MG whose elemental distribution is inhomogeneous.

Table 2. Chemical composition of all films measured by EDX (calibrated with ion beam analysis data).

\begin{tabular}{|c|c|c|c|c|c|c|}
\hline Sample & Zr (at.\%) & Cu (at.\%) & Al (at.\%) & Ni (at.\%) & Ag (at.\%) & O (at. \%) \\
\hline 0 at. $\%$, floating, $60^{\circ} \mathrm{C}$ & 52.4 & 25.4 & 11.3 & 7.1 & - & 3.8 \\
\hline 7.5 at. $\%$, floating, $60^{\circ} \mathrm{C}$ & 50.1 & 23.1 & 10.1 & 6.4 & 7.7 & 2.6 \\
\hline 15 at. $\%$, floating, $60^{\circ} \mathrm{C}$ & 45.9 & 20.7 & 9.0 & 5.8 & 15.1 & 3.5 \\
\hline 30 at. $\%$, floating, $60^{\circ} \mathrm{C}$ & 38.0 & 15.4 & 6.6 & 4.0 & 27.5 & 8.5 \\
\hline 0 at. $\%$, floating, $200^{\circ} \mathrm{C}$ & 53.8 & 24.8 & 11.0 & 6.8 & - & 3.6 \\
\hline 7.5 at. $\%$, floating, $200{ }^{\circ} \mathrm{C}$ & 49.6 & 23.0 & 9.5 & 5.9 & 8.0 & 4.0 \\
\hline 15 at. $\%$, floating, $200{ }^{\circ} \mathrm{C}$ & 46.2 & 20.0 & 8.4 & 5.1 & 15.6 & 4.7 \\
\hline 30 at. $\%$, floating, $200{ }^{\circ} \mathrm{C}$ & 36.9 & 15.3 & 6.6 & 4.0 & 32.6 & 4.6 \\
\hline 0 at. $\%,-80 \mathrm{~V}, 60^{\circ} \mathrm{C}$ & 55.1 & 25.0 & 10.1 & 6.8 & - & 3.0 \\
\hline 7.5 at. $\%,-80 \mathrm{~V}, 60^{\circ} \mathrm{C}$ & 50.6 & 22.5 & 8.6 & 5.9 & 8.4 & 4.0 \\
\hline 15 at. $\%,-80 \mathrm{~V}, 60^{\circ} \mathrm{C}$ & 46.5 & 19.8 & 7.4 & 4.3 & 16.1 & 4.9 \\
\hline 30 at. $\%,-80 \mathrm{~V}, 60^{\circ} \mathrm{C}$ & 37.1 & 14.8 & 6.1 & 3.7 & 32.1 & 6.2 \\
\hline 0 at. $\%,-80 \mathrm{~V}, 200^{\circ} \mathrm{C}$ & 53.7 & 24.8 & 11.1 & 6.6 & - & 3.8 \\
\hline 7.5 at. $\%,-80 \mathrm{~V}, 200^{\circ} \mathrm{C}$ & 52.2 & 22.9 & 8.9 & 6.1 & 5.7 & 4.2 \\
\hline 15 at. $\%,-80 \mathrm{~V}, 200^{\circ} \mathrm{C}$ & 46.7 & 19.6 & 7.5 & 5.2 & 16.9 & 4.1 \\
\hline 30 at. $\%,-80 \mathrm{~V}, 200^{\circ} \mathrm{C}$ & 38.7 & 15.8 & 6.8 & 4.1 & 29.6 & 5.0 \\
\hline
\end{tabular}

Ag has been known to change the local structure of $\mathrm{Cu}$-containing metallic glasses because of the positive heat of mixing of $\mathrm{Ag}$ and $\mathrm{Cu}[33,34]$. However, these changes seem to also depend on the Ag concentration. Gammer et al. reported that even small concentration variations may affect the medium range order of $\mathrm{CuZrAl}(\mathrm{Ag})$ metallic glasses. In fact, they showed that Ag concentrations $>5$ at.\% destabilize the B2 structured clusters [44]. Furthermore, Rajan et al. did not observe any phase separation in the ZrCuAlAg TFMG they produced ( 5 at.\% in Ag) [45]. Therefore, one may expect similar inhomogeneities in elemental distribution as presented in Figure 6 for Ag contents exceeding 5 at.\%.

The electrical resistivities of all films were calculated from measured sheet resistances and thicknesses, and are plotted in Figure 7 as a function of Ag content. STEM and SEM cross-section micrographs of selected samples are also provided. Ag and $\mathrm{Cu}$ exhibit the lowest electrical resistivities among the metals considered herein (Table 3). As the Ag content is increasing, and the $\mathrm{Zr}$ decreasing, the formation of Ag segregations should be evidenced by a drop in the electrical resistivity of the film.

As can be seen from Figure 7, this is true in the case of the films deposited at $200{ }^{\circ} \mathrm{C}$, with and without bias, in which the formation of elongated Ag clusters was observed in cross-sectional images. The resistivity was at its lowest for the $30 \mathrm{at} \% \mathrm{Ag}$ films because of the large volume fraction of Ag segregations.

Despite the presence of Ag segregations on the surface of the films deposited at $60{ }^{\circ} \mathrm{C}$, the resistivity marginally increased with increasing Ag content. The fact that, due to lack of thermal activation, no Ag segregates can form in the bulk of the film may be responsible for the increased resistivity. This is observed for all studied Ag concentrations. Additionally, the films deposited at a substrate bias potential of $-80 \mathrm{~V}$ displayed lower electrical resistivities which may be due to ion bombardment induced densification.

Furthermore, we can conclude that the surface Ag segregations are not affecting the measured resistivities significantly as long as the percolation threshold is not reached. Indeed, films deposited at $60{ }^{\circ} \mathrm{C}$ and the low Ag-content films deposited at $200{ }^{\circ} \mathrm{C}$, exhibit discontinuous Ag segregations on the surface. Depositions at $200{ }^{\circ} \mathrm{C}$ and Ag contents $>15$ at. \%, led to the formation of a continuous polycrystalline Ag layer on the surface. Considering 
that the thicknesses of the formed layers are similar for both 15 and 30 at.\% $\mathrm{Ag}$, the decrease of the resistivity with increasing Ag content is primarily caused by Ag segregations in the bulk of the film.

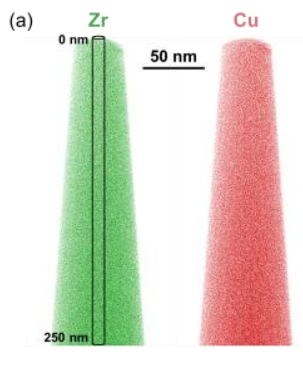

(c)

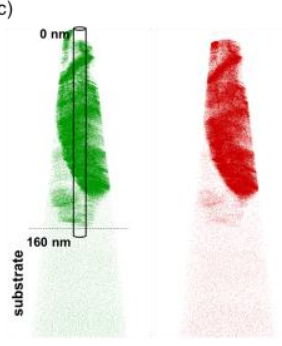

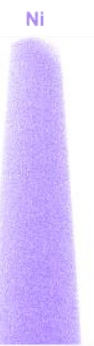

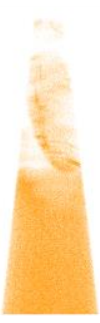

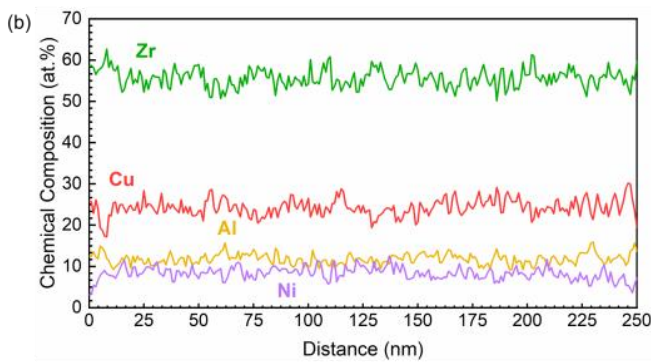

$\mathrm{Ag} \quad(\mathrm{d})$

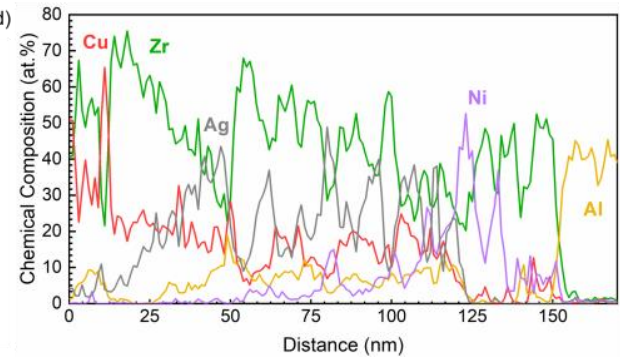

Figure 6. (a) Elemental reconstruction of $\mathrm{Zr}, \mathrm{Cu}, \mathrm{Al}$, and Ni positions, and (b) concentration profile of the cylindrical region indicated in (a), of the pure TFMG, deposited without bias at $60^{\circ} \mathrm{C}$. (c) Reconstruction of $\mathrm{Zr}, \mathrm{Cu}, \mathrm{Al}, \mathrm{Ni}$, and $\mathrm{Ag}$ positions, and (d) concentration profile of the cylindrical region indicated in (c), of the TFMG containing 30 at.\% Ag, deposited without bias at $60{ }^{\circ} \mathrm{C}$.
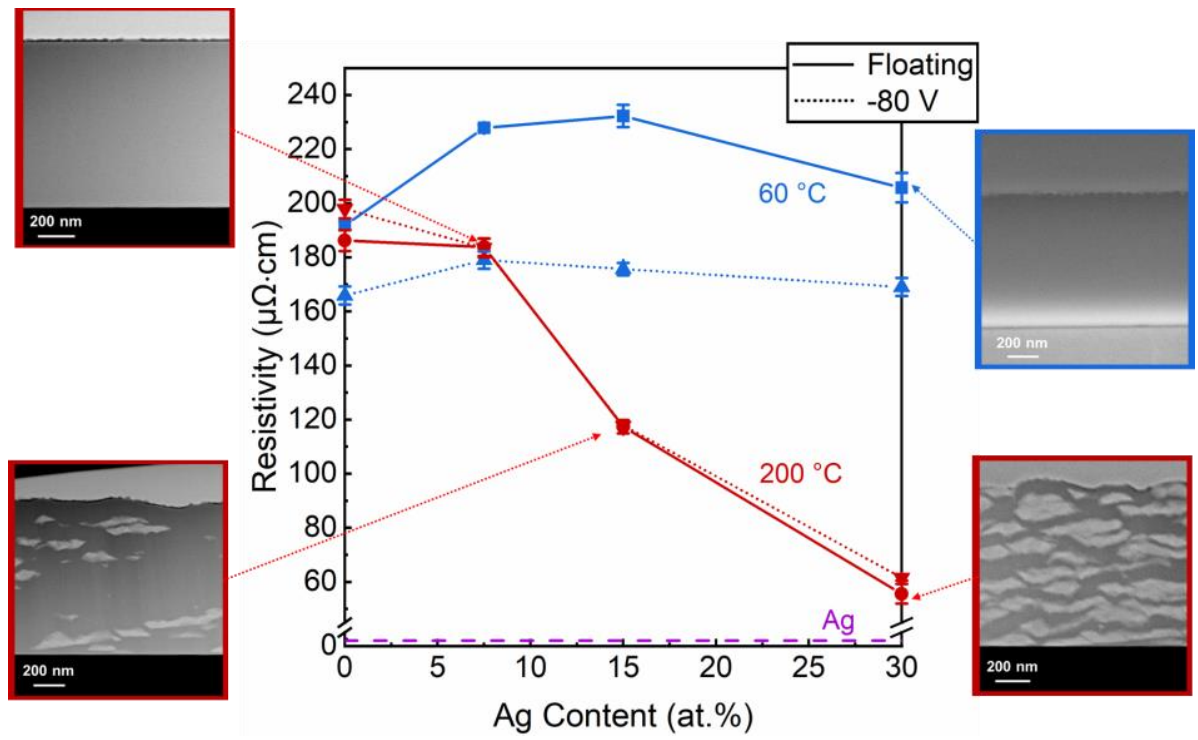

Figure 7. Electrical resistivities of films deposited with (continuous lines) and without (dotted lines) bias at 60 (blue) and $200{ }^{\circ} \mathrm{C}$ (red), for the different Ag contents, accompanied by corresponding cross-section SEM (30 at.\% Ag, floating, $60{ }^{\circ} \mathrm{C}$ ) and STEM images (7.5-30 at.\% Ag, floating, $200{ }^{\circ} \mathrm{C}$ ).

Table 3. Electrical resistivities of metals of interest at $20^{\circ} \mathrm{C}$.

\begin{tabular}{lllccc}
\hline Metal & $\mathbf{Z r}$ & $\mathbf{C u}$ & Al & Ni & Ag \\
\hline Electrical resistivity $(\mu \Omega \cdot \mathrm{cm})$ & 42 & 1.7 & 2.6 & 7 & 1.6 \\
\hline
\end{tabular}

Previously, electrical resistivity has been employed to track phase transitions within $\mathrm{Cr}_{2} \mathrm{AlC}$ [46] and the oxidation behavior of TiN and $\mathrm{V}_{2} \mathrm{AlC}[47,48]$. In this work, we show 
for the first time, that the formation of Ag segregations in sputtered $\mathrm{ZrCuAlNi}$ metallic glass thin films can be tracked by resistivity measurements.

\section{Conclusions}

Ag-containing ZrCuAlNi TFMGs were obtained by hybrid co-sputtering from a Zr$\mathrm{CuAlNi}$ target and an elemental Ag target operated in DCMS and HPPMS, respectively. The Ag contents were $0,7.5,15$, and 30 at.\%, and obtained by varying the $\mathrm{ZrCuAlNi}$ target power. Substrate bias potential and substrate temperature variations were employed in order to determine their effect on the morphology evolution of the films. Despite the chemical affinity between metals and the amorphous nature of the matrix, the segregation mechanisms appear to be similar to those encountered in ceramics matrices.

Most Ag-containing films deposited at $60^{\circ} \mathrm{C}$ exhibited strong surface segregation as Ag NPs were detected on the surfaces of all films except on that of the 7.5 at.\%-containing film deposited without bias. Here scanning electron microscopy image analysis revealed the absence of Ag segregations suggesting a critical metastable Ag solubility of $>7.5$ at. $\%$ at this growth condition. The substrate bias potential influenced the surface mobility of incoming $\mathrm{Ag}$ atoms, as evidenced by the formation of surface segregations at $7.5 \mathrm{at} . \% \mathrm{Ag}$. Increased Ag-contents ( $\geq 15$ at.\%) led to the formation of larger and more numerous $\mathrm{Ag}$ segregations, driven by coalescence and Ostwald ripening. Substrate temperature also had an important role in enhancing surface diffusion and promoting coalescence of $\mathrm{Ag}$ at the surface, as continuous polycrystalline layers were observed on the surface of the films deposited at $200{ }^{\circ} \mathrm{C}$.

Elongated Ag segregations were observed in the bulk of the films deposited at $200{ }^{\circ} \mathrm{C}$, which contained 15 and 30 at.\% of $\mathrm{Ag}$, as a result of thermally enhanced surface diffusion. The MG matrix was still X-ray amorphous and did not present other microstructural features. The formation of metallic Ag segregations observed by cross-sectional microscopy caused a significant decrease in electrical resistivity. In fact, the electrical resistivity was shown to be a useful tool for identifying the presence of conductive Ag segregations in the bulk of the films.

Author Contributions: Conceptualization, M.K.S., J.M.S. and C.A.; methodology, M.K.S. and C.A.; validation, M.K.S., J.M.S. and C.A.; formal analysis, M.K.S., D.N. and M.H.; investigation, M.K.S., D.M.H., S.K.A., D.N., P.J.P. and D.P.; resources, J.M.S. and D.P.; data curation, M.K.S., M.H. and C.A.; writing-original draft preparation, C.A.; writing-review and editing, all authors; visualization, M.K.S., D.N. and C.A.; supervision, J.M.S. and C.A.; project administration, C.A. and D.P.; funding acquisition, C.A. All authors have read and agreed to the published version of the manuscript.

Funding: Excellence Strategy of the Federal Government and the Länder (EXU-JPI044-20), VetenskapsrådetRFI (2017-00646_9), Swedish Foundation for Strategic Research (RIF14-0053).

Institutional Review Board Statement: Not applicable.

Informed Consent Statement: Not applicable.

Data Availability Statement: The data that support the findings of this study are available from the corresponding author, $\mathrm{CA}$, upon request.

Acknowledgments: This work was supported by the Federal Ministry of Education and Research (BMBF) and the Ministry of Culture and Science of the German State of North Rhine-Westphalia (MKW) under the Excellence Strategy of the Federal Government and the Länder (EXU-JPI04420). Support for the operation of the accelerator laboratory in Uppsala by VR-RFI (Contract No. 2017-00646_9) and the Swedish Foundation for Strategic Research (Contract No. RIF14-0053) is gratefully acknowledged.

Conflicts of Interest: The authors declare no conflict of interest. The funders had no role in the design of the study; in the collection, analyses, or interpretation of data; in the writing of the manuscript, or in the decision to publish the results. 


\section{References}

1. Bhat, A.; Budholiya, S.; Raj, S.A.; Sultan, M.T.H.; Hui, D.; Shah, A.U.M.; Safri, S.N.A. Review on nanocomposites based on aerospace applications. Nanotechnol. Rev. 2021, 10, 237-253. [CrossRef]

2. Calderon Velasco, S.; Cavaleiro, A.; Carvalho, S. Functional properties of ceramic-Ag nanocomposite coatings produced by magnetron sputtering. Prog. Mater. Sci. 2016, 84, 158-191. [CrossRef]

3. Guo, J.; Zhao, X.; Herisson De Beauvoir, T.; Seo, J.-H.; Berbano, S.S.; Baker, A.L.; Azina, C.; Randall, C.A. Recent Progress in Applications of the Cold Sintering Process for Ceramic-Polymer Composites. Adv. Funct. Mater. 2018, 28, 1801724. [CrossRef]

4. Ju, H.; Yu, D.; Yu, L.; Ding, N.; Xu, J.; Zhang, X.; Zheng, Y.; Yang, L.; He, X. The influence of Ag contents on the microstructure, mechanical and tribological properties of ZrN-Ag films. Vacuum 2018, 148, 54-61. [CrossRef]

5. Hong, C.; Huan, Y.; Zhang, P.; Zhang, K.; Dai, P. Effect of silver content on the microstructure, thermal stability and mechanical properties of $\mathrm{CrNx} / \mathrm{Ag}$ nanocomposite films. Ceram. Int. 2021, 47, 25324-25336. [CrossRef]

6. Calderón-Jiménez, B.; Johnson, M.E.; Montoro Bustos, A.R.; Murphy, K.E.; Winchester, M.R.; Baudrit, J.R.V. Silver nanoparticles: Technological advances, societal impacts, and metrological challenges. Front. Chem. 2017, 5, 6. [CrossRef]

7. Deshmukh, S.P.; Patil, S.M.; Mullani, S.B.; Delekar, S.D. Silver nanoparticles as an effective disinfectant: A review. Mater. Sci. Eng. C 2019, 97, 954-965. [CrossRef]

8. Cavaleiro, D.; Carvalho, S.; Cavaleiro, A.; Fernandes, F. TiSiN(Ag) films deposited by HiPIMS working in DOMS mode: Effect of Ag content on structure, mechanical properties and thermal stability. Appl. Surf. Sci. 2019, 478, 426-434. [CrossRef]

9. Manninen, N.K.; Galindo, R.E.; Benito, N.; Figueiredo, N.M.; Cavaleiro, A.; Palacio, C.; Carvalho, S. Ag-Ti(C, N)-based coatings for biomedical applications: Influence of silver content on the structural properties. J. Phys. D. Appl. Phys. 2011, $44,375501$. [CrossRef]

10. Domingues, R.P.; Rodrigues, M.S.; Lopes, C.; Pedrosa, P.; Alves, E.; Barradas, N.P.; Borges, J.; Vaz, F. Thin films composed of metal nanoparticles $(\mathrm{Au}, \mathrm{Ag}, \mathrm{Cu})$ dispersed in $\mathrm{AlN}$ : The influence of composition and thermal annealing on the structure and plasmonic response. Thin Solid Films 2019, 676, 12-25. [CrossRef]

11. De Los Arcos, T.; Oelhafen, P.; Aebi, U.; Hefti, A.; Düggelin, M.; Mathys, D.; Guggenheim, R. Preparation and characterization of TiN-Ag nanocomposite films. Vacuum 2002, 67, 463-470. [CrossRef]

12. Folkenant, M.; Nygren, K.; Malinovskis, P.; Palisaitis, J.; Persson, P.O.Å.; Lewin, E.; Jansson, U. Structure and properties of Cr-C/Ag films deposited by magnetron sputtering. Surf. Coat. Technol. 2015, 281, 184-192. [CrossRef]

13. Manninen, N.K.; Galindo, R.E.; Carvalho, S.; Cavaleiro, A. Silver surface segregation in Ag-DLC nanocomposite coatings. Surf. Coat. Technol. 2015, 267, 90-97. [CrossRef]

14. Lopes, C.; Fonseca, P.; Matamá, T.; Gomes, A.; Louro, C.; Paiva, S.; Vaz, F. Protective Ag: TiO2 thin films for pressure sensors in orthopedic prosthesis: The importance of composition, structural and morphological features on the biological response of the coatings. J. Mater. Sci. Mater. Med. 2014, 25, 2069-2081. [CrossRef]

15. Wang, H.; Wang, L.; Wang, X. Structure characterization and antibacterial properties of Ag-DLC films fabricated by dual-targets HiPIMS. Surf. Coat. Technol. 2021, 410, 126967. [CrossRef]

16. Escobar Galindo, R.; Manninen, N.K.; Palacio, C.; Carvalho, S. Advanced surface characterization of silver nanocluster segregation in Ag-TiCN bioactive coatings by RBS, GDOES, and ARXPS. Anal. Bioanal. Chem. 2013, 405, 6259-6269. [CrossRef]

17. Aouadi, S.M.; Bohnhoff, A.; Sodergren, M.; Mihut, D.; Rohde, S.L.; Xu, J.; Mishra, S.R. Tribological investigation of zirconium nitride/silver nanocomposite structures. Surf. Coat. Technol. 2006, 201, 418-422. [CrossRef]

18. Meškinis, S.; Vasiliauskas, A.; Šlapikas, K.; Gudaitis, R.; Andrulevičius, M.; Čiegis, A.; Niaura, G.; Kondrotas, R.; Tamulevičius, S. Bias effects on structure and piezoresistive properties of DLC: AG thin films. Surf. Coat. Technol. 2014, 255, 84-89. [CrossRef]

19. Cloutier, M.; Turgeon, S.; Busby, Y.; Tatoulian, M.; Pireaux, J.J.; Mantovani, D. Controlled Distribution and Clustering of Silver in Ag-DLC Nanocomposite Coatings Using a Hybrid Plasma Approach. ACS Appl. Mater. Interfaces 2016, 8, 21020-21027. [CrossRef]

20. Chu, Y.Y.; Lin, Y.S.; Chang, C.M.; Liu, J.K.; Chen, C.H.; Huang, J.C. Promising antimicrobial capability of thin film metallic glasses. Mater. Sci. Eng. C 2014, 36, 221-225. [CrossRef]

21. Comby-Dassonneville, S.; Venot, T.; Borroto, A.; Longin, E.; Der Loughian, C.; Ter Ovanessian, B.; Leroy, M.A.; Pierson, J.F.; Steyer, P. ZrCuAg Thin-Film Metallic Glasses: Toward Biostatic Durable Advanced Surfaces. ACS Appl. Mater. Interfaces 2021, 13, 17062-17074. [CrossRef]

22. Rajan, S.T.; Arockiarajan, A. Thin film metallic glasses for bioimplants and surgical tools: A review. J. Alloys Compd. 2021, 876, 159939. [CrossRef]

23. Guo, Y.N.; Inoue, A.; Han, Y.; Kong, F.L.; Feng, B.; Zhu, S.L.; Ikuhara, Y. Influence of Ag replacement on the formation and heating-induced phase decomposition of $\mathrm{Zr}_{65} \mathrm{~A}_{17.5} \mathrm{Co}_{27.5-\mathrm{x}} \mathrm{Ag}_{\mathrm{x}}(\mathrm{x}=5$ to 20 at.\%) glassy alloys. J. Alloys Compd. 2019, 783, 545-554. [CrossRef]

24. Yang, Y.J.; Cheng, B.Y.; Jin, Z.S.; Gao, H.X.; Ma, M.Z.; Zhang, X.Y. Crystallization kinetics and mechanical properties of $\mathrm{Zr}_{56} \mathrm{Cu}_{24} \mathrm{~A}_{19} \mathrm{Ni}_{7-\mathrm{x}} \mathrm{Ti}_{4} \mathrm{Ag}_{\mathrm{x}}(\mathrm{x}=$ 0, 1, 3, 5, and 7) metallic glasses. J. Alloys Compd. 2020, 816, 152589. [CrossRef]

25. Mattern, N.; Han, J.H.; Pradeep, K.G.; Kim, K.C.; Park, E.M.; Kim, D.H.; Yokoyama, Y.; Raabe, D.; Eckert, J. Structure of rapidly quenched $\left(\mathrm{Cu}_{0.5} \mathrm{Zr}_{0.5}\right){ }_{100-\mathrm{x}} \mathrm{Ag}_{\mathrm{x}}$ alloys (x=0-40 at.\%). J. Alloys Compd. 2014, 607, 285-290. [CrossRef]

26. Schnabel, V.; Köhler, M.; Evertz, S.; Gamcova, J.; Bednarcik, J.; Music, D.; Raabe, D.; Schneider, J.M. Revealing the relationships between chemistry, topology and stiffness of ultrastrong Co-based metallic glass thin films: A combinatorial approach. Acta Mater. 2016, 107, 213-219. [CrossRef] 
27. Björck, M.; Andersson, G. GenX: An extensible X-ray reflectivity refinement program utilizing differential evolution. J. Appl. Crystallogr. 2007, 40, 1174-1178. [CrossRef]

28. Moro, M.V.; Holeňák, R.; Zendejas Medina, L.; Jansson, U.; Primetzhofer, D. Accurate high-resolution depth profiling of magnetron sputtered transition metal alloy films containing light species: A multi-method approach. Thin Solid Films 2019, 686,137416 . [CrossRef]

29. Janson, M.S. CONTES Instruction Manual; Internal Report; Uppsala University: Uppsala, Sweden, 2004.

30. Mayer, M. SIMNRA, a simulation program for the analysis of NRA, RBS and ERDA. AIP Conf. Proc. 1999, 475, 541-544. [CrossRef]

31. Bönninghoff, N.; Diyatmika, W.; Chu, J.P.; Mráz, S.; Schneider, J.M.; Lin, C.L.; Eriksson, F.; Greczynski, G. ZrCuAlNi thin film metallic glass grown by high power impulse and direct current magnetron sputtering. Surf. Coat. Technol. 2021, 412, 127029. [CrossRef]

32. Emmerlich, J.; Mráz, S.; Snyders, R.; Jiang, K.; Schneider, J.M. The physical reason for the apparently low deposition rate during high-power pulsed magnetron sputtering. Vacuum 2008, 82, 867-870. [CrossRef]

33. Kang, D.H.; Jung, I.H. Critical thermodynamic evaluation and optimization of the Ag- $\mathrm{Zr}, \mathrm{Cu}-\mathrm{Zr}$ and $\mathrm{Ag}-\mathrm{Cu}-\mathrm{Zr}$ systems and its applications to amorphous $\mathrm{Cu}-\mathrm{Zr}-\mathrm{Ag}$ alloys. Intermetallics 2010, 18, 815-833. [CrossRef]

34. $\mathrm{Hu}, \mathrm{Y}$.; Rupert, T.J. Atomistic modeling of interfacial segregation and structural transitions in ternary alloys. J. Mater. Sci. 2019, 54, 3975-3993. [CrossRef]

35. Saeed, U.; Flandorfer, H.; Ipser, H. Lead-free solders: Enthalpies of mixing of liquid alloys in the Ag-Ni and Ag-Ni-Sn systems. J. Mater. Res. 2006, 21, 1294-1304. [CrossRef]

36. Adhikari, D.; Singh, B.P.; Jha, I.S. Structural and energetic asymmetry in liquid Ag-Al alloys. Phys. Chem. Liq. 2010, 48, 787-796. [CrossRef]

37. Agraval, P.; Dreval, L.; Turchanin, M.; Storchak-Fedyuk, A.; Artyukh, L.; Velikanova, T. Enthalpy of mixing of liquid Ni-Ti-Zr alloys at 1873 K. J. Chem. Thermodyn. 2017, 106, 309-316. [CrossRef]

38. Yadav, S.K.; Lamichhane, S.; Jha, L.N.; Adhikari, N.P.; Adhikari, D. Mixing behaviour of Ni-Al melt at 1873 K. Phys. Chem. Liq. 2016, 54, 370-383. [CrossRef]

39. Agraval, P.; Turchanin, M.; Dreval, L.; Vodopyanova, A. Mixing enthalpy of liquid Cu-Hf-Ni alloys at 1873 K. J. Therm. Anal. Calorim. 2017, 128, 1753-1763. [CrossRef]

40. Mulligan, C.P.; Papi, P.A.; Gall, D. Ag transport in CrN-Ag nanocomposite coatings. Thin Solid Films 2012, 520, 6774-6779. [CrossRef]

41. Hu, J.J.; Muratore, C.; Voevodin, A.A. Silver diffusion and high-temperature lubrication mechanisms of YSZ-Ag-Mo based nanocomposite coatings. Compos. Sci. Technol. 2007, 67, 336-347. [CrossRef]

42. Incerti, L.; Rota, A.; Ballestrazzi, A.; Gualtieri, E.; Valeri, S. Ag surface diffusion and out-of-bulk segregation in CrN-Ag nano-composite coatings. J. Nanosci. Nanotechnol. 2011, 11, 9260-9266. [CrossRef]

43. Oh, J.C.; Ohkubo, T.; Kim, Y.C.; Fleury, E.; Hono, K. Phase separation in Cu43Zr43Al7Ag 7 bulk metallic glass. Scr. Mater. 2005, 53, 165-169. [CrossRef]

44. Gammer, C.; Escher, B.; Ebner, C.; Minor, A.M.; Karnthaler, H.P.; Eckert, J.; Pauly, S.; Rentenberger, C. Influence of the Ag concentration on the medium-range order in a CuZrAlAg bulk metallic glass. Sci. Rep. 2017, 7, 44903. [CrossRef]

45. Rajan, S.T.; Nanda Kumar, A.K.; Subramanian, B. Nanocrystallization in magnetron sputtered Zr-Cu-Al-Ag thin film metallic glasses. CrystEngComm 2014, 16, 2835-2844. [CrossRef]

46. Stelzer, B.; Chen, X.; Bliem, P.; Hans, M.; Völker, B.; Sahu, R.; Scheu, C.; Primetzhofer, D.; Schneider, J.M. Remote Tracking of Phase Changes in Cr2AlC Thin Films by In-situ Resistivity Measurements. Sci. Rep. 2019, 9, 8266. [CrossRef] [PubMed]

47. Stelzer, B.; Momma, M.; Schneider, J.M. Autonomously Self-Reporting Hard Coatings: Tracking the Temporal Oxidation Behavior of TiN by In Situ Sheet Resistance Measurements. Adv. Funct. Mater. 2020, 30, 2000146. [CrossRef]

48. Azina, C.; Mráz, S.; Greczynski, G.; Hans, M.; Primetzhofer, D.; Schneider, J.M.; Eklund, P. Oxidation behaviour of V2AlC MAX phase coatings. J. Eur. Ceram. Soc. 2020, 40, 4436-4444. [CrossRef] 